\title{
Emprise des champs d'anacarde sur les forêts et savanes en milieu paysan autour du parc national de Comoé
}

\author{
Charles Yao SANGNE*, Issouf BAMBA, Bruno Kouassi KPANGUI, \\ Apollinaire Kouassi KOUAKOU et Yao Sadaiou Sabas BARIMA
}

Université Jean Lorougnon Guédé, Unité de Formation de Recherche en Environnement, BP 150 Daloa, Côte d'Ivoire.

*Corresponding author; E-mail: y_sangne@yahoo.fr, Tel: 0022547969378.

\section{RESUME}

A la faveur de la culture de l'anacarde, savanes et forêts des zones Nord de la Côte d'Ivoire sont transformées en de vastes vergers d'anacardiers. Ces travaux visent à analyser les modifications du paysage dans les espaces ruraux au Sud-Ouest du Parc National de Comoé (PNC), patrimoine mondial de 1'Unesco. De façon spécifique, il s'est agi de: (i) cartographier à partir de trois images satellitaires Landsat acquises respectivement les 29/01/2002, 02/12/2006 et 23/02/2014, l'occupation du sol des espaces ruraux au Sud-Ouest du parc national de Comoé et de (ii) suivre la dynamique des différents types d'occupation du sol de 2002 à 2014. Les résultats ont révélé que les surfaces occupées par la culture de l'anacarde dans la zone couverte par cette étude sont passées de 11743 hectares en 2002 à 29872 hectares en 2014 (soit plus de 160\%), tandis que les surfaces des forêts (galeries forestières et forêts denses sèches) ont fortement régressé et sont passées de 26 242 hectares en 2002 à 6133 hectares en 2014 (soit -76\%). Si la dynamique actuelle du paysage se poursuit, le PNC risque à moyen terme d'être objet de convoitise par les populations rurales, ce qui fragiliserait sa préservation et sa conservation.

(C) 2019 International Formulae Group. All rights reserved

Mots clés: Végétation, Culture de l'anacarde, Télédétection, Cartographie, Dynamique du paysage, Côte d'Ivoire.

\section{Reach of cashew fields on forests and savannas in a rural environment around the National Park of Comoé}

\begin{abstract}
Owing to the cultivation of cashew nuts, savannas and forests in the northern parts of Côte d'Ivoire are transformed into vast orchards of cashew trees. This work aims to analyze landscape changes in the rural areas in the South-West of the Comoé National Park (CNP) which is known as Unesco World Heritage. Specifically, it consisted of: (i) mapping from three Landsat satellite images acquired respectively on 2002/01/29, 2006/12/02 and 2014/02/23, the land use of the South-West of Comoé National Park, and (ii) monitor the dynamics of different types of land use from 2002 to 2014 . The results revealed that the areas covered by the
\end{abstract}


cashew crop in the study zone increased from 11,743 hectares in 2002 to 29,872 hectares in 2014 (ie more than $160 \%$ ), while forest areas (galleries forest and dry dense forests) declined sharply from 26,242 hectares in 2002 to 6,133 hectares in 2014 (ie - 76\%). If the current dynamics of the landscape continues, the CNP could be coveted by the rural populations, in the medium term, so that would weaken its preservation and conservation.

(C) 2019 International Formulae Group. All rights reserved

Keywords: Vegetation, Cashew crop, Remote sensing, Cartography, Landscape Dynamics, Côte d'Ivoire.

\section{INTRODUCTION}

$\mathrm{Au}$ fil des années, l'anacarde (Anacardium occidentale L.) est devenu une spéculation à laquelle s'adonnent la plupart des pays d'Afrique subsaharienne. L'intérêt pour cette spéculation a suscité plusieurs travaux scientifiques (Djaha et al., 2012 ; Balogoun et al., 2014 ; Batamoussi et al., 2017, Seydou et al., 2017). Outre la caractérisation des agrosystèmes à base d'anacarde, la plupart de ces travaux visent à améliorer le rendement des vergers à la production par la mise à disposition auprès des producteurs de plants sélectionnés et d'itinéraire technique plus adaptés. Ainsi, l'Afrique de l'Ouest est devenue, ces dernières années, la première région productrice d'anacarde au monde (Audouin et Gonin, 2014). En Côte d'Ivoire, depuis 1970, l'anacardier est devenu, une culture fruitière de rente dont la production ne fait qu'augmenter (Djaha, 2012). Estimée à 16 000 tonnes en 1994, puis à 78000 tonnes en 2001 (Dugue et al., 2003), la production ivoirienne de noix de cajou a atteint 380000 tonnes en 2010 (Soro, 2012). La campagne 2015 - 2016, a estimé cette production à près de 700000 tonnes (Koulibaly et al., 2016), faisant de la Côte d'Ivoire le premier producteur mondial devant l'Inde, le Brésil et le Viet-Nam. Pour les régions du Centre et du Nord de la Côte d'Ivoire, la culture de l'anacarde est devenue une des principales sources de revenue des paysans. En effet, cette culture fruitière de rente, peu exigeante des conditions climatiques (la pluviométrie relativement faible dans les régions Centre et Nord du pays) et édaphiques, est soutenue par des cours meilleurs ces dernières années (le prix du kilogramme bord-champ de noix de cajou pour la campagne 2016-2017, a été fixé 440 Fcfa). Ainsi, les superficies des surfaces d'anacardiers augmentent chaque année. Selon les analystes de l'African Cashew Alliance (2011), l'accroissement des superficies d'anacardiers africains, pourrait porter la croissance de la production mondiale de noix de cajou à près de $34 \%$ au cours de la période 2010 - 2020.

En Côte d'Ivoire, savanes et forêts des zones Nord du pays sont en train d'être transformées en de vastes surfaces agricoles pour la culture de l'anacarde. La forte propension des populations vers cette spéculation a entraîné une forte réduction des surfaces agricoles des espaces ruraux. Cette étude vise à analyser les modifications du paysage dans les espaces ruraux au Sud-Ouest du Parc National de Comoé (PNC), patrimoine mondial de l'Unesco. Elle est fondée sur les hypothèses suivantes :

- la forte propension des paysans pour la culture de l'anacarde risquerait à court ou long terme d'entraîner une monopolisation des terres agricoles au détriment des cultures vivrières ;

- l'épuisement des terres agricoles (savanes et forêts) alentours du parc national de Comoé risquerait l'isolement du PNC, réserve de Biosphère ;

- la culture de l'anacarde devenue une manne financière importante pour les communautés locales ne fragiliserait-elle pas à la longue le statut du parc national de Comoé (Patrimoine mondial de l'Unesco), dont les terres seraient convoitées pour l'agriculture de subsistance? 
De façon spécifique, il s'est agi, sur la base du traitement et de l'interprétation de trois images satellitaires Landsat acquises respectivement les 29/01/2002, 02/12/2006 et 23/02/2014, puis validation après des campagnes de terrain, de cartographier d'une part l'occupation du sol du Sud-Ouest du parc national de Comoé et de sa périphérie en 2002, 2006 et 2014 et, d'autre part, de suivre la dynamique de ces différents types d'occupation de 2002 à 2014.

\section{MATÉRIEL ET MÉTHODES Milieu d'étude}

Le PNC est situé au Nord-Est de la Côte d'Ivoire, entre les latitudes $8^{\circ} 30^{\prime}$ et $9^{\circ} 37^{\prime}$ Nord et les longitudes $3^{\circ} 07^{\prime}$ et $4^{\circ} 26^{\prime}$ Ouest (Figure 1). Il est la plus grande aire protégée de la Côte d'Ivoire avec une superficie totale de 1149450 hectares. La végétation de cette aire protégée et de ses espaces ruraux, est dominée par les formations savanicoles dans lesquelles, on retrouve plusieurs fragments de forêts de tailles variables. La contigüité presque permanente des savanes avec ces fragments de forêts imprime à la végétation du PNC et de sa zone périphérique un faciès qui s'apparente à celui d'une "brousse tigrée", très remarquable pendant la saison sèche.

\section{Matériel et méthodes}

Trois images satellitaires Landsat ont servi à la réalisation de ces travaux. Il s'agit de Landsat ETM+ du 29/01/2002, Landsat ETM+ du 02/12/2006 et Landsat OLI du 23/02/2014. Ces images ont toutes la même résolution $(30 \mathrm{~m})$ et ont été téléchargées sur le site

www.glcfapp.glcf.umd.edu.8080/esdi/index.js p.

De chacune de ces trois (3) images déjà géoréférencées, la zone couverte par l'étude (Sud-Ouest du parc national de Comoé) a été extraite. Cette zone d'intérêt correspond à une fenêtre de 1837 colonnes sur 1725 lignes. En effet, compte tenu de la superficie du parc de Comoé (1 149450 hectares), des moyens financiers assez restreints et surtout des données de base grâce à certains travaux notamment (Hennenberg et al., 2005; Koulibaly, 2008; Kouassi et al., 2014; Koulibaly et al., 2016), il n'était pas possible de prendre en compte l'intégralité du PNC.

Pour mettre en évidence les différents types d'occupation du sol, des indices de végétation ont été utilisés et ont permis de séparer les surfaces couvertes par une végétation de celles des sols nus et des plans d'eau. Il s'est agi des indices tels que : Normalized Difference Vegetation Index (NDVI) ou indice de végétation normalisé, adapté pour mettre en évidence les surfaces couvertes par une végétation généralement photosynthétiquement active,

- Brightness index (BI) ou indice de brillance des sols, adapté pour caractériser les sols nus sans couvert végétal important,

- Wetness Index (WI) ou Indice d'Humidité adapté pour caractériser l'humidité au niveau des végétaux et des sols.

Outre les indices, les compositions colorées $(\mathrm{etm}+457$ et etm+347) ont été indispensables pour séparer en fonction de leur réflectance les différents types d'occupations qui se partagent le Sud-Ouest $\mathrm{du}$ PNC et sa zone périphérique. Ces compositions colorées ont mis en évidence différentes colorations, sur la base desquelles, des points géographiques ont été sélectionnés puis enregistrés dans un GPS pour la campagne de terrain. En moyenne 5 points par types de coloration ont été enregistrés et visités lors de la campagne de terrain.

Les différents traitements couplés aux observations (types de cultures, pistes, techniques culturales, localités, recouvrement des forêts, etc.) recueillies lors de la campagne de terrain, ont conduit à la classification dirigée des trois images. La méthode de classification du maximum de vraisemblance est celle qui a servi à exécuter cette classification. 


\section{Analyse statistiques}

Pour évaluer la classification, un test statistique basé sur la réalisation d'une matrice de confusion pour chacune des images classifiées a été réalisé. Ce test donne la précision cartographique affichée pour chaque classe, la précision cartographique globale, la valeur de l'indice de Kappa. Cet indice exprime en effet, la réduction proportionnelle de l'erreur obtenue par une classification comparée à l'erreur obtenue avec une classification pseudo-aléatoire (Girard et Girard, 1999). Un indice de Kappa s'échelonnant de 0,61 à 0,80 peut être estimé satisfaisant (Santos, 2015). Par ailleurs, les changements au sein des différentes classes d'occupation du sol ont été vérifiés à l'aide d'une matrice de transition (Bell, 1974; Turner, 1989; Schlaepfer, 2002; Bamba,
2010). Cette dernière a permis de mettre en évidence les proportions d'affectation (ou de transformation) d'un type d'occupation du sol donné en 2002 en un autre type en 2006, de 2006 à 2014 et de 2002 à 2014.

Afin de mieux rendre compte de l'évolution des surfaces des champs d'anacarde durant ces différentes périodes (2002-2006; 2006-2014 et 2002-2014), un masque a été construit pour extraire soit la partie de la zone d'étude contenue à l'intérieur du PNC, soit la partie de la zone d'étude située en dehors de la limite sud du PNC. La largeur du "buffer" contenant la zone périphérique du Sud-Ouest du PNC est d'environ $5 \mathrm{~km}$.

Tous ces traitements radiométriques et cartographiques ont été réalisés à partir des logiciels ENVI 4.7 et QGIS.

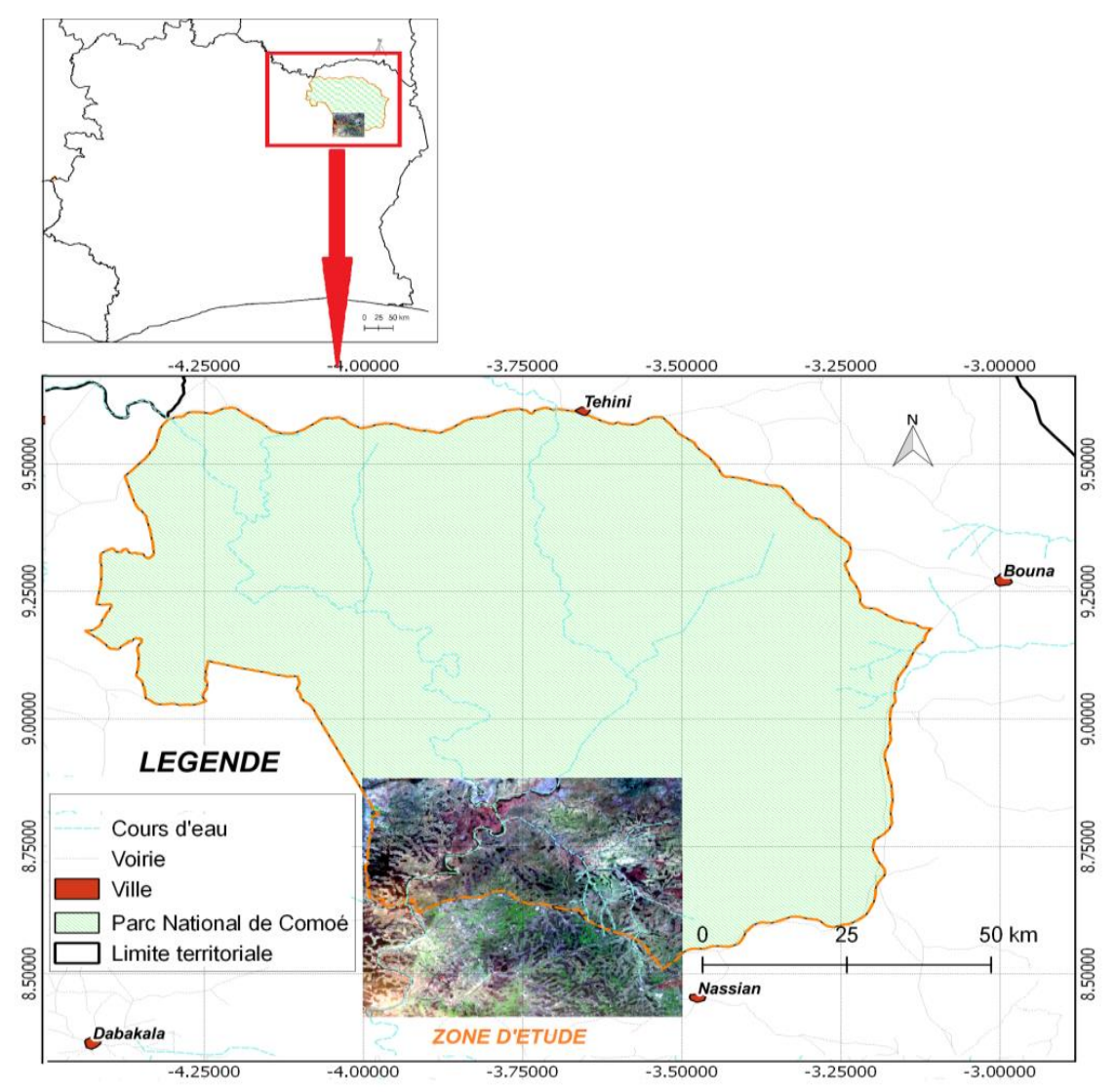

Figure 1 : Localisation de la zone d'étude dans le Sud-Ouest du Parc National de la Comoé. 


\section{RÉSULTATS}

La démarche méthodologique suévoquée a permis d'aboutir aux résultats suivants :

\section{Cartographie des types d'occupation de sol}

Les traitements réalisés sur les trois images ont permis d'identifier neuf (09) classes d'occupation du sol à savoir : Brûlis, Savanes arbustives avec un couvert herbacé plus important et des ligneux peu denses dont la taille n'excède guère 5 mètres de hauteur, Savanes arborées (boisées), Galeries forestières et/ou Forêts rupicoles, Forêts claires, Forêts denses, Plantations d'anacarde, Sols nus et/ou Localités et la classe Eau représentée par le fleuve Comoé (Figure 2). Les précisions cartographiques globales et indices de Kappa respectifs à l'issu de la classification des différentes images (Tableau 1) sont satisfaisantes. Toutefois, des confusions (Tableaux 2a, 2b et 2c) existent entre certaines classes. Ainsi, pour l'image de 2006, de fortes confusions ont été observées entre les savanes arbustives et boisées $(41,14 \%)$; entre forêt dense et forêt galerie $(41,58 \%)$; forêt claire et savane boisée $(17,50 \%)$, puis entre plantations d'anacarde et Galeries forestières $(43,75 \%)$ durant la période de crise (2006).

Pour l'image de 2002, elles ont été observées entre Brûlis et Savanes arbustives $(27,61 \%)$, puis entre Brûlis et Savanes boisées $(13,80 \%)$.

Quant à l'image de 2014, les confusions ont surtout été observées entre les classes Sols nus et Savanes arbustives $(22,33 \%)$.

En considérant les différentes cartes produites, toutes les classes d'occupation du sol existent sur chacune des cartes. Cependant, de 2002 à 2014, on observe une recomposition progressive du paysage spatial par les différentes classes. En effet, sur la carte de 2002, on peut apercevoir un dense réseau d'îlots forestiers dont la présence est très remarquable à l'extérieur du parc. Les cultures d'anacarde y sont présentes, mais leurs surfaces se résument à des taches plus ou moins importantes laissant toujours place aux fragments forestiers. Le long du fleuve Comoé, les forêts rupicoles forment un continuum plus ou moins épais. L'image de 2006 met en évidence le début de l'installation d'un front pionnier très actif pour la culture de l'anacarde dans le domaine rural et dont les îlots forestiers constituent la cible principale. L'image de 2014 plus récente, laisse apparaître une modification plus profonde du paysage environnant du parc national de Comoé. Cette modification (transformation) est surtout le fait des vergers d'anacardiers qui occupent désormais des surfaces plus importantes matérialisées par des taches plus compactes et l'apparition de plusieurs espaces dénudés ou sols nus.

\section{Occupation du sol sur la période 2002 à 2014}

Pour une meilleure compréhension et compte tenu des confusions entre les différentes classes de Savanes et entre les classes de Forêts, il s'est avéré opportun de fusionner les classes de Savanes en une seule classe. Il en a été de même pour les classes de Forêts (Figure 3).

De 2002 à 2014, les classes Savanes et Forêts demeurent toujours les plus importantes dans l'occupation de l'espace de la zone soumise à étude. Les classes Sols nus /Localités et Champs d'anacarde sont les moins représentées. Cependant, on note que les surfaces de ces dernières ont connu une augmentation progressive de 2002 à 2014 (Figure 3).

\section{Tendances évolutives des différents types d'occupation du sol à l'extérieur du PNC}

Pour comprendre la dynamique d'expansion de la culture d'anacarde dans l'espace du parc national de Comoé, il était nécessaire d'examiner les tendances évolutives des différents types d'occupation du sol dans la zone périphérique à l'extérieur de ses limites conventionnelles. De 2002 à 2006, les forêts denses évoluent positivement 
avec les forêts claires, les surfaces des cultures d'anacardes et les sols nus. Au contraire, les surfaces des forêts claires, des savanes arbustives et des plans d'eau ont connu de fortes régressions au cours de la même période. En ce qui concerne la période 2006 - 2014, cette période est caractérisée par une régression des surfaces des forêts denses, forêts claires, forêts galeries, savanes arborées et plans d'eau; tandis que la tendance est à la hausse pour les surfaces des cultures d'anacarde, des sols nus/habitats et des savanes arbustives (Figure 4).
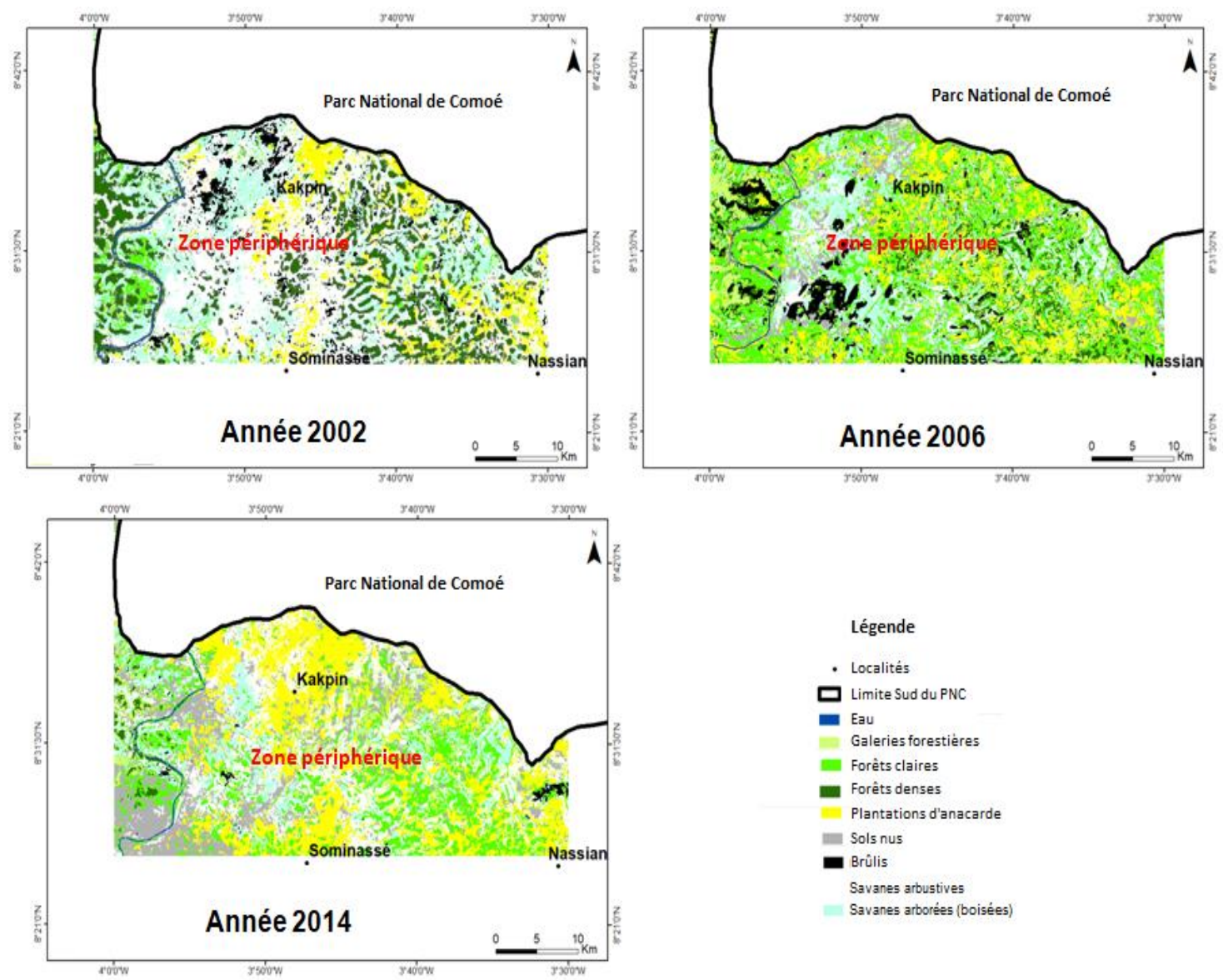

Figure 2: Cartes d'occupation du sol du Sud-Ouest du Parc National de Comoé en 2002, 2006 et 2014.

Tableau 1: Paramètres de validation des cartes produites.

\begin{tabular}{llll}
\hline Année & $\mathbf{2 0 0 2}$ & $\mathbf{2 0 0 6}$ & $\mathbf{2 0 1 4}$ \\
\hline Précision globale & 87,1727 & 73,032 & 82,13 \\
\hline Indice de Kappa & 0,8524 & 0,6848 & 0,7958 \\
\hline
\end{tabular}


Tableau 2 : Matrice de confusion à l'issu de la classification des différentes images satellitaires. 2a : Matrice de confusion de 2002.

\begin{tabular}{llllllllll}
\hline & Brûlis & Sav. arbt & Sav. arbo_ & Fo_cl & Fo_gal_rip & Fo_dense & Eau & Sol_nu & Plt_ana \\
\hline Brûlis & 58,59 & 1,74 & 0 & 0 & 0 & 0 & 0 & 0 & 0 \\
\hline Sav; arbt. & 27,61 & 88,35 & 4,65 & 0 & 0 & 0 & 0 & 2,68 & 0 \\
\hline Sav. arbo. & 13,80 & 7,65 & 92,25 & 2,32 & 0 & 0 & 0 & 0 & 0 \\
\hline Fo_cl & 0 & 0 & 3,10 & 90,95 & 3,92 & 0,75 & 0 & 1,34 & 3,86 \\
\hline Fo_gal_rip & 0 & 0 & 0 & 1,86 & 84,05 & 4,38 & 0 & 0 & 1,93 \\
\hline Fo_dense & 0 & 0 & 0 & 2,78 & 2,48 & 94,74 & 6,80 & 0 & 0 \\
\hline Eau & 0,13 & 0 & 0 & 0 & 0 & 0,13 & 93,20 & 0 & 0 \\
\hline Sol_nu & 0 & 0 & 0 & 0,23 & 0 & 0 & 0 & 95,97 & 0 \\
\hline Plt_ana & 0 & 2,26 & 0 & 1,86 & 9,54 & 0 & 0 & 0 & 94,22 \\
\hline
\end{tabular}

2b : Matrice de confusion de 2006.

\begin{tabular}{llllllllll}
\hline & Brûlis & Sav. arbt. & Sav. arbo. & Fo_cl & Fo_gal_rip & Fo_dense & Eau & Sol_nu & PIt_ana \\
\hline Brûlis & 98,85 & 0 & 0 & 0 & 0 & 0 & 0,78 & 0 & 0 \\
\hline Sav. arbt & 0,10 & 50,41 & 2,29 & 0,12 & 0 & 0 & 0 & 0 & 0 \\
\hline Sav. arbo. & 0 & 41,14 & 78,56 & 17,50 & 0 & 0 & 0 & 0,39 & 0 \\
\hline Fo_cl & 0,1 & 0,57 & 18,73 & 50,48 & 0,46 & 0,17 & 0 & 0,39 & 0 \\
\hline Fo_gal_rip & 0 & 0 & 0 & 6,07 & 77,43 & 41,58 & 0,78 & 0 & 43,75 \\
\hline Fo_dense & 0,29 & 0 & 0 & 25,36 & 8,97 & 58,25 & 0 & 0 & 0 \\
\hline Eau & 0 & 0 & 0 & 0 & 0 & 0 & 98,44 & 0 & 0 \\
\hline Sol_nu & 0,67 & 7,37 & 0,42 & 0,24 & 0,08 & 0 & 0 & 91,86 & 0,69 \\
\hline Plt_ana & 0 & 0,51 & 0 & 0,24 & 13,07 & 0 & 0 & 7,36 & 55,56 \\
\hline
\end{tabular}

2c : Matrice de confusion de 2014.

\begin{tabular}{llllllllll}
\hline Class & Brûlis & Sav. arbt. & Sav. arbo. & Fo_cl & Fo_gal_rip & Fo_dense & Eau & Sol_nu & PIt_ana \\
\hline Brûlis & 96,18 & 0 & 1,11 & 0 & 0,19 & 0 & 0,45 & 0 & 0 \\
\hline Sav. arbt. & 0,32 & 82,88 & 7,75 & 0 & 0 & 0 & 0 & 22,33 & 0 \\
\hline Sav. arbo. & 3,50 & 0 & 57,56 & 11,09 & 0,19 & 0 & 0,45 & 0 & 0 \\
\hline Fo_cl & 0 & 0 & 30,63 & 77,43 & 7,36 & 17,13 & 0 & 0 & 0,3 \\
\hline Fo_gal_rip & 0 & 0 & 0 & 0,59 & 87,21 & 7,32 & 0,45 & 0 & 0,61 \\
\hline Fo_dense & 0 & 0 & 0 & 0 & 1,16 & 75,55 & 1,36 & 0 & 0 \\
\hline Eau & 0 & 0 & 0 & 0 & 0 & 0 & 97,29 & 0 & 0 \\
\hline Sol_nu & 0 & 17,12 & 1,85 & 0 & 0 & 0 & 0 & 71,84 & 0 \\
\hline Plt_ana & 0 & 0 & 1,11 & 10,89 & 3,88 & 0 & 0 & 5,78 & 99,09
\end{tabular}

Sav.arbt: Savanes arbustives ; Sav.arbo.: Savanes arborées ; Fo.cl. : Forêts claires ; Fo.gal.rip.: Forêts galeries ou ripicoles ; Fo.dense : Forêts denses ; Plt.ana.: Plantations d'anarcadiers. 
C. Y. SANGNE et al. /Int. J. Biol. Chem. Sci. 13(2): 662-675, 2019

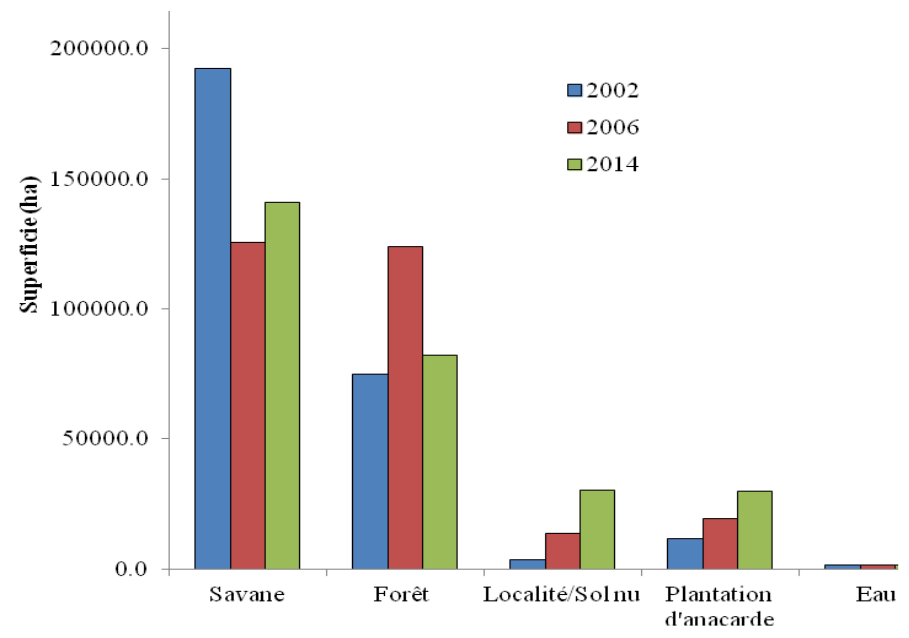

Figure 3 : Dynamique des types d'occupation du sol de 2002 à 2014.

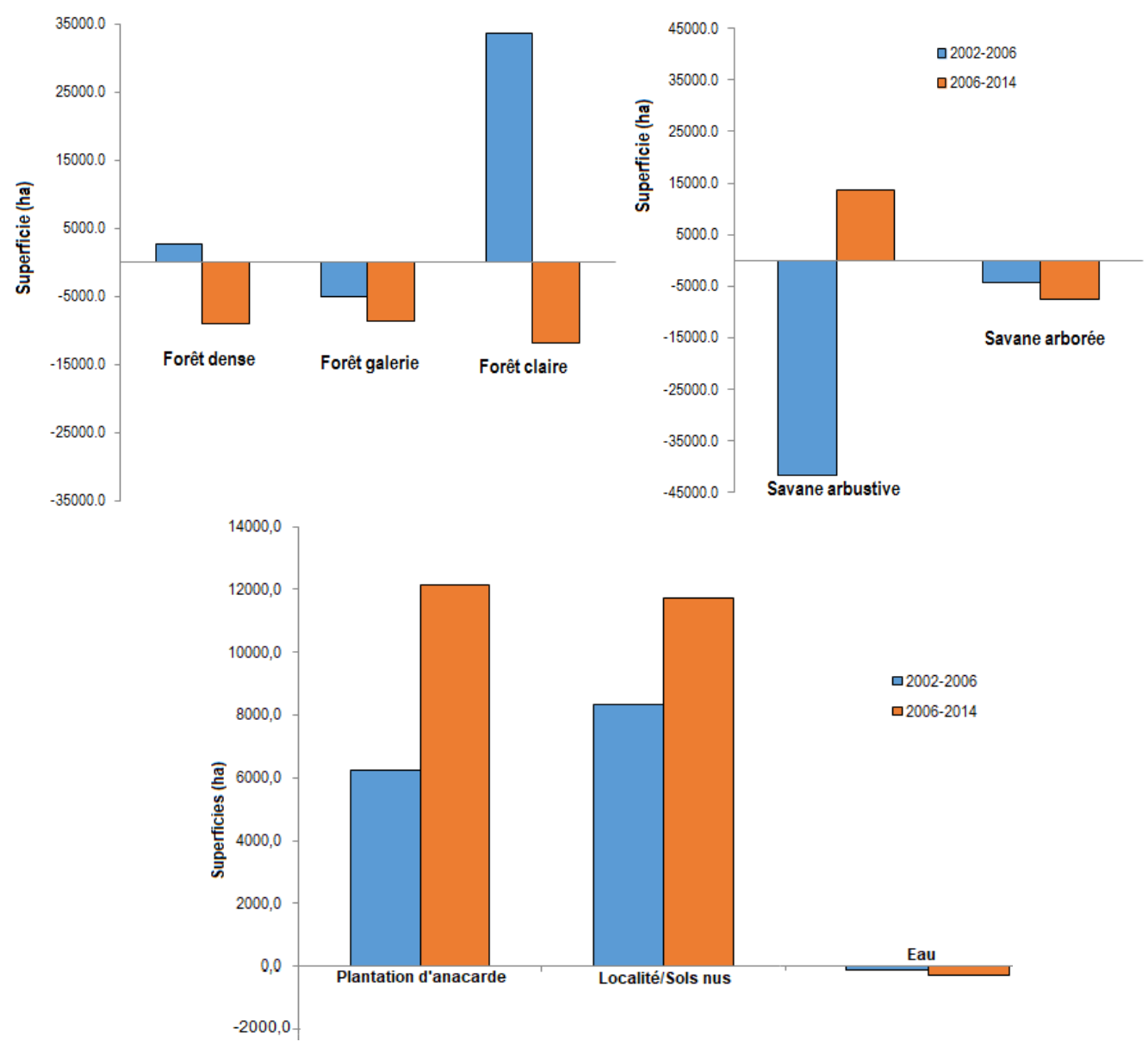

Figure 4: Tendances évolutives des différentes classes d'occupation du sol à la périphérie du PNC. 


\section{DISCUSSION}

\section{Dynamique des types d'occupation du sol à la périphérie du PNC de 2002 à 2014}

$\mathrm{La}$ dynamique des différents types d'occupation du sol à la périphérie du PNC est gouvernée (dominée) par les activités agricoles dont les impacts relativement modérés entre 2002 et 2006, se sont fortement intensifiés de 2006 à 2014. Ce front pionnier très actif se manifeste par une augmentation progressive puis significative des surfaces des cultures d'anacardiers. Ces surfaces sont passées d'un peu plus de 11200 ha à environ 17500 ha entre 2002 et 2006 (soit une augmentation de plus de 55\%) puis à plus de 29600 ha (soit environ $70 \%$ ) en 2014. Cette dynamique révèle que les forêts avaient tendance à se reconstituer entre 2002 et 2006. Cette tendance à la reconstitution coïncide avec le début de la crise politico-militaire déclenchée en 2002 et la partition du pays, qui a entraîné dès les premiers instants un départ massif des populations vers le sud du pays sous contrôle gouvernemental et offrant relativement plus de sécurité. Malheureusement, la tendance à la reconstitution des espaces va s'estomper, les forces basées dans la moitié Nord du pays opposées aux forces gouvernementales basées dans le sud vont se livrer à un pillage des ressources naturelles. De ce fait plusieurs fragments forestiers aussi bien des espaces ruraux qu'à l'intérieur du PNC vont être investis impunément pour leurs bois d'œuvre, leur richesse faunique, etc. Ces faits ont eu pour conséquence, l'inscription du parc national de Comoé sur la liste du Patrimoine mondial en péril par l'Unesco en 2003 (Koueita et al., 2018). A l'exploitation forestière s'est ajoutée plus tard, à partir de 2006, un retour progressif des populations qui s'accompagne d'une reprise des activités agricoles. Ces activités vont s'intensifier davantage entre 2006 et 2014, avec pour conséquences la conversion des savanes et forêts en champs d'anacardiers. Ces faits corroborent certains travaux notamment ceux de Koulibaly et al. (2016) qui ont montré que la dynamique de l'occupation du sol à l'extérieur du Sud du PNC est orientée vers la création de plantations d'anacardiers. Ces dernières se sont installées au détriment des îlots forestiers. Or, selon Akobi et al. (2018), la végétation conditionne en grande partie l'état des sols, de la faune et d'autres paramètres de l'existence des êtres vivants dont l'homme son prédateur. De telles modifications du paysage pourraient donc être préjudiciables aux populations riveraines $\mathrm{du}$ PNC qui en sont les principaux acteurs.

\section{Monopolisation des terres par la culture de l'anacarde, source d'insécurité alimentaire et de conflits fonciers}

La régression des forêts et savanes mises en exergue sur les fonds de cartes dans cette étude s'est faite au profit des champs d'anacardiers (Koulibaly et al., 2016). L'ampleur de ces vergers d'anacardiers qui occupent désormais les espaces forestiers, puis les savanes, suscite plusieurs inquiétudes dans la région. En effet, la propension de bon nombre d'agriculteurs pour la culture de l'anacarde va entraîner un rétrécissement considérable des surfaces destinées aux cultures vivrières. Il faut noter que les populations des localités du Nord-Est (Lobi en particulier) de la Côte d'Ivoire, culturellement ont pour aliment de base l'igname dont elles produisent de fortes quantités aussi bien pour leur propre consommation que pour approvisionner les marchés de l'intérieur du pays. Selon les travaux de certains auteurs, six (6) principaux bassins de production de l'igname de variétés diverses ont été identifiés en Côte d'Ivoire dont deux (2) sont représentés par les bassins Bouna-Bondoukou, Kong- 
Dabakala (Sekou et al., 2006). Ces bassins sont localisés dans les espaces ruraux du parc national de Comoé. La dynamique actuelle du paysage orientée vers la création de champs d'anacardiers pourrait donc conduire inexorablement à un rétrécissement ou à une conversion des terres arables destinées à l'agriculture de subsistance. Il va sans dire que les populations riveraines du PNC risquent d'être confrontées à une situation d'insécurité alimentaire. Dans un tel contexte, elles devront, pour se nourrir, acheter du vivrier sur les marchés d'autres régions productrices du pays. Il convient de signaler que la région de Korhogo, autrefois grande productrice de vivriers (igname notamment), de nos jours est obligée recourir à d'autres régions du pays pour approvisionner son marché (Sekou et al., 2006).

Par ailleurs, dans le contexte de la situation socio-politique actuelle de la Côte d'Ivoire, en tenant compte des conflits liés au foncier très récurrents dans le sud du pays du fait de la cacaoculture, la culture de l'anacarde vue son ampleur, à la longue peut être à l'origine de tensions communautaires et conflits entre communautés locales dans l'occupation des espaces ruraux autour du parc national de Comoé et partant, dans tous les espaces ruraux du Nord de la Côte d'Ivoire. Dans cette perspective, il est à craindre à moyen ou à long terme le déplacement des conflits récurrents dans le Sud du pays vers le Nord pour l'anacarde. Cette situation n'épargnerait guère les aires protégées (PNC en particulier) et les forêts classées des zones sub-soudaniennes et soudaniennes de la Côte d'Ivoire, et avec elles les populations animales et végétales qu'elles abritent (. En effet, la quasi-totalité des forêts classées du Sud de la Côte d'Ivoire sont entièrement ou partiellement occupées par des cacaoculteurs (N'Da, 2007 ; N'Da et al., 2008 ; Kouakou et al., 2015 ; Sangne et al., 2015 ; Barima et al., 2016). De même, certaines aires protégées du pays notamment le parc national de la Marahoué, le parc national du Mont Péko, le parc national d'Azagny, la réserve le Mont Nimba, connaissent une situation préoccupante du fait de la cacaoculture (Goné Bi et al., 2013 ; Koffi et al., 2015); d'où une remise en cause certaine de la politique de préservation de l'environnement prônée par les décideurs, et des engagements pris par l'Etat de Côte d'Ivoire sur la préservation de l'environnement devant les grandes instances internationales (Conference of parties (COP) 21, 22, 23, ... ; ONU ; REDD+, FAO, etc.) est à craindre.

\section{Menace pour la protection et la conservation de la diversité biologique et risque de conflits Homme-Animaux}

La dynamique du paysage dans le domaine rural du parc national de Comoé, caractérisée par une transformation rapide des forêts et savanes en vergers d'anacardiers constitue nul doute une menace pour la protection et la conservation de la diversité biologique qu'abrite cette aire protégée. Les habitats de plusieurs populations d'insectes (les abeilles par exemple) et de microfaunes, maillons essentiels des systèmes écologiques terrestres sont souvent détruits. En effet, selon Kouassi et al. (2018), l'agriculture dont la conséquence est la déforestation ne favorise pas l'installation des colonies d'abeilles pour la production du miel. Par ailleurs, la connexion entre les fragments de forêts dans les espaces ruraux et les forêts domaniales à la périphérie du PNC est menacée par l'intensification de la culture de l'anacarde. Cette situation, combinée aux activités de braconnage dans les limites du PNC, ne sont pas de nature à favoriser le déplacement des grands mammifères facteurs de dispersion des semences des plantes (cas de l'éléphant), de 
brassage entre populations animales et surtout de richesses spécifiques. Il est alors à craindre à moyen ou long terme un musellement, voire un isolement du PNC, et avec lui toutes les populations d'animaux qu'il comporte du fait de l'absence d'un corridor écologique pouvant garantir le mouvement des grands mammifères. Par ailleurs, nonobstant la superficie relativement importante du PNC estimée à près de 1150000 hectares, il n'est pas exclu qu'une modification quelconque à long terme des facteurs écologiques de cette aire protégée ne contraigne les populations animales qui y vivent à rechercher d'autres espaces offrant des meilleures conditions (disponibilité de nourriture, d'eau, de sécurité, ...). Dans ces conditions, l'absence de véritable corridor de coexistence et par conséquent, l'isolement du PNC risque outre de prédisposer les populations riveraines à des conflits avec les grands mammifères parmi lesquels les fauves, mais de les exacerber davantage, tous les espaces ruraux et "domaniaux" étant occupés par les activités humaines.

\section{Conclusion}

Les objectifs assignés à cette étude étaient de rendre compte, après traitement et interprétation de trois images satellitaires Landsat acquises respectivement les 29/01/2002, 02/12/2006 et 23/02/2014, sur des fonds de cartes les différentes modifications intervenues dans les paysages ruraux à la périphérie du parc national de Comoé (PNC), puis de suivre la dynamique des différents types d'occupation de ces espaces de 2002 à 2014. Il ressort de ces travaux que la dynamique du paysage à la périphérie du parc national de Comoé est caractérisée par une augmentation des surfaces des vergers d'anacardiers. Ces surfaces sont passées d'environ 11200 hectares en 2002 à près de
17500 hectares en 2006 (soit plus de 55\%), puis à plus de 29600 hectares en 2014 (soit plus de 160\%). Au contraire, les surfaces des forêts (galeries forestières et forêts denses sèches) ont fortement régressé et sont passées de 26242 hectares en 2002 à 6133 hectares en 2014 (soit une baisse de plus de -76\%). Ces modifications du paysage se sont faites au détriment des reliques forestières d'abord puis des savanes. Si l'exploitation des terres du domaine rural pour la culture de l'anacarde se poursuit au rythme actuel, le parc national de Comoé risque l'isolement à moyen terme, à l'instar du parc national de Taï dans le SudOuest de la Côte d'Ivoire. Une convoitise des terres à l'intérieur de $\mathrm{PNC}$, ce qui fragiliserait sa conservation et sa protection, est donc à craindre.

\section{CONFLIT D'INTÉRÊTS}

Les auteurs déclarent qu'ils n'ont aucun conflit d'intérêts.

\section{CONTRIBUTIONS DES AUTEURS}

BYSS a conçu et supervisé le projet de recherche; KKA a aidé à la collecte des données sur le terrain; BI et KKB ont fait les différents traitements statistiques et produit les cartes ; SYC a analysé les données et rédigé le manuscrit ; BI, BYSS, KKA, KKB et SYC ont corrigé et approuvé la version finale du manuscrit.

\section{REMERCIEMENTS}

La présente étude a bénéficié du financement $\mathrm{du}$ Fonds Français pour l'Environnement Mondial (FFEM) dans le cadre du projet C2D - D2PCPCI avec l'appui technique de l'Institut de Recherche pour le Développement (IRD). L'étude a été possible grâce à la collaboration de l'Office Ivoirien des Parcs et Réserves (OIPR) qui a autorisé l'accès au Parc National de la Comoé et des 
gestionnaires de la Station de Recherche en Ecologie.

\section{RÉFÉRENCES}

ACA. 2011. African Cashew Alliance, Annual Report 2011. Accra, Ghana, ACA, 16 p.

Akobi KI, Amoussou E, Yabi I, Boko M. 2018. Historique de l'évolution anthropique et dynamique du couvert végétal dans la commune de Bantè au Bénin. Int. J. Biol. Chem. Sci., 12(1): 180-194. http://www.ifgdg.org/

Audouin S, Gonin A. 2014. L'anacarde : produit de la globalisation, moteur de la territorialisation, l'exemple du Sud du Burkina Faso. EchoGéo, 29. DOI: https://doi.org/10.3917/eg.431.0035

Balogoun I, Saïdou A, Ahoton EL, Amadjl GI, Ahohuendo CB, Adebo IB, Babatounde S, Chougourou D, Adoukonou-Sagbadja H, Ahanchede A. 2014. Caractérisation des systèmes de production à base d'anacardier dans les principales zones de culture au Benin. Agronomie Africaine, 26 (1) : 9- 22.

Bamba I. 2010. Anthropisation et dynamique spatio-temporelle de paysages forestiers en République Démocratique du Congo, Thèse unique de doctorat, Université Libre de Bruxelle, Belgique, 181 p.

Barima YSS, Kouakou KA, Kouakou ATM, Bamba I, Sangne YC, Godron M, Andrieu J, Bogaert J. 2016. Cocoa crops are destroying the forest reserves of the classified forest of Haut-Sassandra (Ivory Coast). Global Ecology and Conservation, 8: 85-98. DOI: https://doi.org/10.1016/j.gecco.2016.08.0 09.

Batamoussi MH, Tokore Orou Mere SBJ, Moussa I, Karami OM, Amanoudo M-J,
Lawson RG. 2017. Contribution à l'amélioration du taux de réussite du greffage de l'anacardier (Anacardium occidentale) en pépinière dans la commune de Parakou au Nord-Bénin. Int. J. Biol. Chem. Sci., 11(5): 2270-2276.

Bell EJ. 1974. Markov analysis of land use change: an application of stochastic process to remotely sensed data. SocioEconomic Planning Sciences, 8: 311316. DOI : https://doi.org/10.1016/00380121(74)90034-2

Djaha J-BA, N'da Adopo AA, Koffi EK, Ballo CK, Coulibaly M. 2012. Croissance et aptitude au greffage de deux génotypes d'anacardier (Anacardium occidentale L.) élites utilisés comme porte-greffe en Côte d'Ivoire. Int. J. Biol. Chem. Sci., 6(4): 1453-1466.

http://ajol.info/index.php/ijbcs

Dugue P, Fahiraman RK, Gnagandjomon K. 2003. Gestion des ressources naturelles et évolution des systèmes de production agricoles des savanes de Côte d'Ivoire : conséquences pour l'élaboration des politiques agricoles. Savanes africaines : des espaces en mutation, des acteurs face à de nouveaux défis. Actes du colloque, Garoua, Cameroun. Cirad-Prasac.

Girard MC, Girard CM. 1999. Traitement des Données de Télédétection. Dunod: Paris, 529 p.

Goné Bi ZB, Kouame D, Kone I, Adou Yao CY. 2013. Diversité végétale et valeur de conservation pour la Biodiversité du Parc National du Mont Péko, une aire protégée, menacée de disparition en Côte d'Ivoire. Journal of Applied Biosciences, 71(1): 5753-5762. $\quad$ DOI: http://dx.doi.org/10.4314/jab.v71i1.9882 0 
Hennenberg KJ, Goetze D, Kouamé L, Orthmann B, Porembski S. 2005. Border and ecotone detection by vegetation composition along forest-savanna transects in Ivory Coast. Journal of Vegetation Science, 16: 301-310. DOI: https://doi.org/10.1111/j.16541103.2005.tb02368.x

Koffi KAD, Adou Yao CY, Vroh Bi TA, Gnagbo A. 2015. Diversités floristiques et structurale des espaces anciennement cultivés du Parc National D'azagny (Sud de la Côte d'Ivoire). European Journal of Scientific Research, 134(4): 415-427 http://www.europeanjournalofscientificre search.com

Kouadio BH, Tahoux MT, Biemi J. 2006. Apport de la télédétection et d'un système d'informations géographiques (SIG) à la gestion intégrée et participative des aires protégées en Côte d'Ivoire : application au parc national de comoé (réserve de biosphère) : Rapport d'étude; programme UNESCO/MAB pour Jeunes Scientifiques 2006, 60 pages.

Kouakou ATM, Barima YSS, Kouakou KA, Kouamé NF, Yatty KJ. 2015. Forest dynamics in armed conflicts period in classified forest of Haut-Sassandra (Ivory Coast). American Journal of Life Sciences, $\quad 3(5): \quad 375-382$. http://www.sciencepublishinggroup.com/ j/ajls. DOI: 10.11648/j.ajls.

Kouassi DF, Ouattara D, Coulibaly S., N'Guessan KE. 2018. La cueillette, la production et la commercialisation du miel dans le Département de Katiola (Centre-Nord, Côte d'Ivoire). Int. J. Biol. Chem. Sci., 12(5): 2212-2225.

Kouassi KE, Sangne YC, Dibi NH. 2014. Typologie de la végétation par une approche de signature spectrale dans le sud du parc national de la Comoé (NordEst Côte d'Ivoire). European Scientific Journal, 10(36).

Koueita MKK, Diomandé M, Brou AN. 2018. La problématique de gestion du parc national de la Comoé (PNC) en Côte d'Ivoire, entre la survie des populations riveraines et la conservation de la biodiversité. European Scientific Journal, 14: 391-411. DOI : http://dx.doi.org/10.19044/esj.2018.v14n $35 \mathrm{p} 391$

Koulibaly A. 2008. Caractéristiques de la végétation et dynamique de la régénération, sous l'influence de l'utilisation des terres, dans des mosaïques forêts-savanes, des régions de la Réserve de Lamto et du Parc National de la Comoé, en Côte d'Ivoire. Doctorat de thèse unique de l'Université de Cocody-Abidjan, 216p.

Koulibaly A, Akédrin N, Massé D, Ibrahim K, Dossahoua T, Ralf B, Yatty JK. 2016. Conséquences de la culture de l'anacardier (Anacardium occidentale L.) sur les caractéristiques de la végétation dans la région du Parc National de la Comoé (Côte d'Ivoire). International Journal of Innovation and Applied Studies, 17(4): 1416-1426. http://www.ijias.issr-journals.org/

N'Da DH. 2007. Étude et suivi par télédétection et système d'information géographique d'une aire protégée soumise aux pressions, Thèse de Doctorat unique, Université de CocodyAbidjan, 174 p.

N'Da HD, N'Guessan EK, Wajda ME, Affian K. 2008. Apport de la télédétection au suivi de la déforestation dans le Parc National de la Marahoué (Côte d'Tvoire). Bulletin-Société Française de 
Photogrammétrie et de

Télédétection, 8(1) : 17-34.

Sangne YC, Barima YSS, Bamba I, N'Doumé

CTA. 2015. Dynamique forestière postconflits armés de la Forêt classée du Haut-Sassandra (Côte d'Ivoire). VertigO, 15(3) : 1-18.

https://id.erudit.org/iderudit/1035879ar.

Santos F. 2015. Le kappa de Cohen : un outil de mesure de l'accord inter-juges sur des caractères qualitatifs. CNRS, UMR 5199 PACEA. http://www.pacea.ubordeaux1.fr/IMG/pdf/Kappa_Cohen.pdf . 5 pages. Consulté le 18/02/2018.

Schlaepfer R. 2002. Analyse de la dynamique $\mathrm{du}$ paysage. Fiche d'enseignement 4.2, Laboratoire de Gestion des Écosystèmes, École polytechnique de Lausanne, Suisse.

Sekou D, Moustapha T, Mahyao A. 2006. Commercialisation de l'igname en Côte d'Ivoire: état actuel et perspectives d'évolution. Cahiers Agricultures, 15(3) : $273-277$.

Seydou N, Mohamed MC, Ndiaye O. et Malaïny D. 2017. Influence de la flore ligneuse associée dans la production des parcs à Anacardium occidentale L. dans la communauté rurale de Djibanar (Casamance/Sénégal). Int. J. Biol. Chem. Sci., 11(2): 585-596.

Soro D. 2012. Couplage de procédés membranaires pour la clarification et la concentration du jus de pomme de cajou : performances et impacts sur la qualité des produits. Thèse de doctorat, Centre international d'études supérieures en sciences agronomiques; spécialité génie des procédés ; Montpellier Supagro. 135 p.

Turner MG. 1989. Landscape ecology: the effect of pattern on process. Annual Review of Ecological System, 20: 171197. 